\title{
Reproducing color images with embedded metallic patterns
}

\author{
Roger D. Hersch ${ }^{1}$, Fabien Collaud $^{1}$, Patrick Emmel ${ }^{2}$ \\ ${ }^{1}$ Ecole Polytechnique Fédérale de Lausanne (EPFL), Switzerland \\ ${ }^{2}$ Clariant International, Muttenz, Switzerland
}

\begin{abstract}
By combining a metallic ink and standard inks, one may create printed images having a dynamic appearance: an image viewed under specular reflection may be considerably different from the same image viewed under non-specular reflection. Patterns which are either dark or hidden become highlighted under specular reflection, yielding interesting visual effects. To create such images, one needs to be able to reproduce at non-specular reflection angles the same colors, by standard inks alone or in combination with a metallic ink. Accurate color prediction models need to be established which model the underlying physical phenomena in a consistent manner. To meet this challenge, we propose two models, one for predicting the reflection spectra of standard inks on coated paper and one for predicting the reflection spectra of a combination of standard inks and a metallic ink. They are enhancements of the classical Clapper-Yule model which models optical dot gain of halftone prints by taking into account lateral scattering within the paper bulk and multiple internal reflections. The models we propose also take into account physical dot gain and ink spreading for standard inks as well as the low reflectance of metallic inks at nonspecular reflection angles and the poor adherence of standard inks printed on top of a metallic ink (trapping effect). These models open the way towards color separation of images to be reproduced by combining a metallic ink and standard inks. Several designs printed on an offset press demonstrate their applicability and their benefits for high-end design and security applications.
\end{abstract}

Keywords: Color reproduction, metallic ink printing, color prediction model, dot gain, ink spreading, trapping,

\section{Introduction}

In contrast to standard inks, metallic inks have the particularity of incorporating a strong specular reflection component. At nonspecular angles however, metallic inks do not reflect much light, i.e. they behave as if they were strongly absorbing inks. We are interested in enriching the design space of artists by providing the tools to allow the incorporation of metallic patterns into images printed with standard cyan, magenta, and yellow inks. Target applications include the creation of high-end designs and security documents.

Traditional techniques exist for creating metallic-looking images. Often, a metallic sheet, either gold or silver, is engraved with appropriately designed patterns, and at some places, overprinted with color inks. Thanks to different engravings of the metallic sheet, specific patterns stand out at different observation angles (examples: http://www.creatiftransfert.com/original2.html, http://www.whshop.co.uk/acatalog/Dufex_Prints.html).

Proc. SIGGRAPH 2003, ACM Trans. on Graphics, Vol. 22, No.3, 427-436
We would like to explore to what extent metallic effects can be produced when combining standard and metallic inks within the same printed image. More specifically, we are interested in enhancing at specular reflection angles the visibility of microstructure patterns embedded into a color image. We are also interested in offering support for designs making use of the inversion effect, i.e. transitions between darkness at non-specular reflection angles and highlights at specular reflection angles. Finally, we would like to be able to hide a ghost pattern at non-specular angles and make it apparent at specular angles.

These goals can be reached if we can replace the coverages of standard cyan $(c)$, magenta $(m)$ and yellow $(y)$ inks used in offset printing by an appropriate coverage of an achromatic metallic ink, called silver $(s)$, and adapted coverages of standard cyan $\left(c_{s}\right)$, magenta $\left(m_{s}\right)$ and yellow $\left(y_{s}\right)$ inks printed on top of the metallic ink. Under non-specular light reflection, a printed cmy patch should not be distinguishable from its corresponding printed $s c_{s} m$ ${ }_{s} y_{s}$ patch. Since silver is dark at non specular reflection angles, the transformation from $c m y$ to $s c_{s} m_{s} y_{s}$ can be conceptualized as a special kind of undercolor removal.

In order to be able to find for given coverages cmy of standard inks the corresponding coverages $s c_{s} m_{s} y_{s}$ of the superposition of silver and standard inks, we need accurate color printing prediction models, both for standard inks and for the combination of a metallic ink (silver) and standard inks.

Due to the large number of phenomena which influence the reflection spectrum of a color patch printed at different coverages of inks on a given substrate, the establishment of an accurate model capable of predicting the color of offset prints remains a difficult task. Furthermore, one needs to take into account phenomena which are specific to metallic ink printing such as the interaction of light and the metallic ink, the strongly reduced amount of a standard ink that can be deposited over a metallic ink (trapping effect) and the intriguing fact that a standard ink printed over a metallic ink raises the metallic ink's reflection spectrum at non-specular reflection angles.

We address these problems by generalizing in an original way the well-known Clapper-Yule model [Clapper and Yule 1953] and taking specifically into account phenomena such as ink spreading when printing one ink over a second ink, trapping of a standard ink printed over a metallic ink, and the raised metallic ink reflection spectrum phenomenon.

With the establishment of a prediction model capable of predicting the spectrum of standard inks and of the combination of a metallic ink and standard inks, we can construct a lookup table for creating the correspondence between given coverages of cyan, magenta, and yellow inks and corresponding coverages of silver, cyan, magenta and yellow. Such a 3D lookup table allows then by tri-linear interpolation to very quickly replace standard inks by a combination of silver and standard inks.

In section 2, we briefly review existing color prediction models. In section 3, we introduce an enhanced prediction model for predicting the reflectance spectra of standard inks. In section 4 , we adapt the enhanced prediction model in order to take into account the phenomena that are specific to printing with metallic inks. In section 5, we describe issues related to the creation of the calibration tables needed for printing with combinations of a metallic and standard inks. In section 6, we show images with embedded metallic ink patterns synthesized according to the proposed model. 
These images illustrate how embedded microstructures can be enhanced by a metallic ink, how visually interesting effects are produced with patterns alternating at different viewing angles between highlight and dark and how patterns can be hidden at nonspecular reflection angles and made apparent at specular reflection angles. Section 7 draws the conclusions.

\section{Related work}

Existing approaches for printing with metallic inks rely on simple undercolor removal techniques. A metallic ink has an assigned c'm'y' equivalent, which is subtracted from the original cmy quantities in order to yield the amounts of $c_{s} m_{s} y_{s}$ to be printed on top of the metallic ink [Williamson et. al. 1994]. However, since the combination of ink layers is not really subtractive, this method cannot ensure a high reproduction fidelity. To ensure a high reproduction fidelity, one needs to rely on a scientific model predicting the spectra (or the colors) of printed ink patches. Establishing a spectral prediction model valid for printing both with standard inks and with a combination of a metallic ink and standard inks represents a considerable challenge.

Many different phenomena influence the reflection spectrum of a color patch printed on a given substrate (e.g. a coated paper) or on top of a metallic ink. We need to take into account the surface (Fresnel) reflection at the interface between the air and the coated paper, the light scattering and reflection within the substrate (paper bulk), the internal (Fresnel) reflections at the interface between the coated paper and the air, possibly the scattering of light by the color pigments, and in the case of a metallic ink, reflection and absorption of light by the metallic ink layer. The establishment of accurate and experimentally verified prediction models remains an active topic of research [Wyble and Berns 2000].

In early printing prediction models, the term "dot gain" encompasses both the physical dot gain (the enlargement of the printed dot) and the optical dot gain due to the lateral propagation of light (scattering within the paper bulk and internal reflections at the interface between paper and air). The Neugebauer model predicts the CIE-XYZ color coordinates (also called tri-stimulus values) of a color halftone patch as the sum of the color coordinates of their individual colorants weighted by their fractional area coverages $a_{i}$ [Demichel 1924]. By replacing the color coordinates of colorants by their respective reflection spectra $R_{i}$, one obtains the spectral Neugebauer equations giving the predicted reflection spectra $R(\lambda)$ of printed color patches.

$$
R(\lambda)=\sum_{i} a_{i} \cdot R_{i}(\lambda)
$$

In the case of independently printed cyan, magenta and yellow inks of respective coverages $c, m, y$, the fractional area coverages of the individual colorants are closely approximated by the Demichel [1924] equations which give the probability of a point to be located within a given colorant area, [Wyble and Berns 2000].

$$
\begin{array}{ll}
\text { white: } & a_{w}=(1-c) \cdot(1-m) \cdot(1-y) \\
\text { cyan: } & a_{c}=c \cdot(1-m) \cdot(1-y) \\
\text { magenta: } & a_{m}=(1-c) \cdot m \cdot(1-y) \\
\text { yellow: } & a_{y}=(1-c) \cdot(1-m) \cdot y \\
\text { red: } & a_{r}=(1-c) \cdot m \cdot y \\
\text { green: } & a_{g}=c \cdot(1-m) \cdot y \\
\text { blue: } & a_{b}=c \cdot m \cdot(1-y) \\
\text { black: } & a_{k}=c \cdot m \cdot y
\end{array}
$$

Since the Neugebauer model neither takes explicitly into account the lateral propagation of light within the paper bulk nor the internal reflections (Fresnel reflections) at the coated paper - air interface, its predictions are not accurate [Kang 1994]. Yule and Nielsen [1951] modeled the non-linear relationship between colorant reflection spectra and predicted reflectance by a power func- tion, whose exponent $n$ is fitted according to a limited set of measured patch reflectances :

$$
R_{t o t}(\lambda)=\left(\sum_{i} a_{i} \cdot R_{i}(\lambda)^{\frac{1}{n}}\right)^{n}
$$

The Clapper-Yule model, described in detail in section 3, models the internal reflections at the coated paper - air interface and assumes that lateral light propagation due to light scattering within the paper bulk is large compared with the period of the halftones. Therefore, the probability of light to exit from a given colorant is set equal to the colorant's fractional surface coverage.

Rogers [2000] generalized Clapper-Yule model models lateral scattering within the paper as a point spread function and deduces the probabilities that light entering through a colorant $n$ emerges from the coated paper through a colorant $m$, possibly traversing, due to multiple reflections, further intermediate colorants. This theoretical model seems convincing, but its adequacy for predicting the spectra of color offset patches needs to be verified.

Within the framework of their work on the reproduction of color images by custom inks, Stollnitz et. al. [1998] predict the reflection spectra of solid colorants by using Kubelka's [1954] layering model for combining the paper layer and the ink layers and by applying Saunderson's [1942] correction taking into account multiple reflections at the interface between the paper coating and the air as well as at the interface between the paper coating and the paper bulk. Halftone color predictions are deduced from solid colorant colors by extending the Neugebauer equations in order to take dot gain and trapping into account.

The approach we follow relies on successive enhancements of the basic Clapper-Yule model. The benefits of each of these enhancements is verified by comparing measured halftone patch spectra and predicted spectra, for 729 patches, produced by generating all combinations of inks at nominal coverages $0 \%, 12.5 \%$, $25 \%, 37.5 \%, 50 \%, 62.5 \%, 75 \%, 87.5 \%$ and $100 \%$. We quantify the visual quality of color halftone patch predictions by converting measured and predicted spectra first to CIE-XYZ and then to CIELAB [Kang 1997, pp. 8-12]. The Euclidian distance $\Delta E$ in CIELAB space gives a measure of the visually perceived distance between measured and predicted spectra. For deriving successive model improvements, we observe the predicted and measured spectra of patches where prediction and measurement disagree (large $\Delta E$ value), try to give an explanation of the underlying phenomena (e.g. ink spreading) and propose an appropriate modeling step.

The measurements are carried out with a photospectrometer having a $45^{\circ} / 0^{\circ}$ geometry, i.e. a light source illuminating the printed sample at an angle of $45^{\circ}$ and a sensor capturing the reflected spectrum at $0^{\circ}$, i.e. normal to the printed sample.

\section{An enhanced Clapper-Yule based spectral color prediction model}

Among the different existing basic color prediction models [Kang 1994], only the Clapper-Yule model takes simultaneously into account halftone patterns and multiple internal reflections occurring at the interface between the coated paper and the air.

For introducing the Clapper-Yule model, we consider a single halftone ink layer with a fractional surface coverage $a$ printed on a coated paper substrate (Figure 1). Incident light has the probability $a$ of reaching the paper substrate by passing through the ink (transmittance $t(\lambda))$ and a probability $(1-a)$ of reaching the substrate without traversing the ink layer. Since $r_{s}$ is the surface reflection at the air-paper coating interface, only portion $\left(1-r_{s}\right)$ actually enters the coated paper. The light reaching the paper substrate is reduced by a factor $\left(1-r_{s}\right)(1-a+a t)$. It is diffusely reflected by the paper substrate according to the paper substrate reflectance $r_{g}(\lambda)$. Travelling upwards, it traverses the coated paper with a portion $a$ traversing the ink and a portion 1- $a$ traversing an area free of ink. It is 
reflected at the coated paper - air interface according to reflection factor $r_{i}$ (Fresnel reflection). A part (1- $\left.r_{i}\right)$ of the light exits. At the first exit, the spectral attenuation of the incident light is therefore $\left(1-r_{s}\right) \mathrm{r}_{\mathrm{g}}\left(1-r_{i}\right)(1-a+a t)^{2}$. The part reflected at the coated paper-air interface travels downward, is diffusely reflected by the paper and travels upwards again. At the second exit, the spectral attenuation is $\left(1-r_{s}\right) \cdot r_{g} \cdot\left(1-r_{i}\right) \cdot(1-a+a \cdot t)^{2} \cdot\left(r_{i} \cdot r_{g} \cdot\left(1-a+a \cdot t^{2}\right)\right)$.

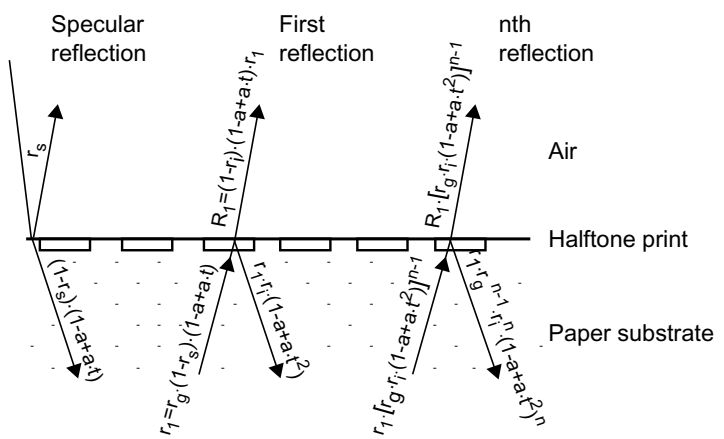

Figure 1: Attenuation of light by multiple reflections on a halftone printed patch

With $K$ giving the fraction of specular reflected light reaching the photospectrometer ${ }^{1}$, and by considering the light emerging after 0 , $1,2, \ldots, n-1$ internal reflections, we obtain the reflection spectrum

$$
\begin{aligned}
R(\lambda)= & K \cdot r_{s}+\left(\left(1-r_{s}\right) \cdot\left(1-r_{i}\right) \cdot r_{g} \cdot(1-a+a \cdot t)^{2}\right) \cdot\left(1+\left(r_{i} \cdot r_{g} \cdot\left(1-a+a \cdot t^{2}\right)\right)\right. \\
& +\left(r_{i} \cdot r_{g} \cdot\left(1-a+a \cdot t^{2}\right)\right)^{2}+\ldots+\left(r_{i} \cdot r_{g} \cdot\left(a+a \cdot t^{2}\right)\right)^{n-1}
\end{aligned}
$$

For an infinite number of emergences, we obtain

$$
R(\lambda)=K \cdot r_{s}+\frac{\left(1-r_{s}\right) \cdot r_{g} \cdot\left(1-r_{i}\right) \cdot(1-a+a \cdot t)^{2}}{1-r_{g} \cdot r_{i} \cdot\left(1-a+a \cdot t^{2}\right)}
$$

In the case of paper printed with 3 inks such as cyan, magenta and yellow, the coverages of the resulting 8 basic colorants, i.e. white ${ }^{2}$, cyan, magenta, yellow, red, green, blue and black are obtained according to the Demichel equations (eq. 2). By inserting the relative amounts of colorants $a_{i}$ and their transmittances $t_{i}$ in equation 4 , we obtain for the predicted reflectance of a color patch printed with combinations of cyan, magenta and yellow inks

$$
R(\lambda)=K \cdot r_{s}+\frac{\left(1-r_{s}\right) \cdot r_{g} \cdot\left(1-r_{i}\right) \cdot\left(\sum_{j=1}^{8} a_{j} \cdot t_{j}\right)^{2}}{1-r_{g} \cdot r_{i} \cdot \sum_{j=1}^{8} a_{j} \cdot t_{j}^{2}}
$$

Both the specular reflection $r_{s}$ and the internal reflection $r_{i}$ depend on the refraction indices of the air $\left(n_{1}=1\right)$ and of the coated paper $\left(n_{2}=1.5\right)$, independently of whether the considered surface is white or printed (the ink penetrates the coated paper surface). According to the Fresnel equations [Hecht 1974, Chapter 3], for collimated light at an incident angle of $45^{\circ}$, the specular reflection factor is $r_{s}=0.05$. With light diffusely reflected by the paper (Lambert radiator), according to [Judd 1942], the internal reflection factor is $r_{i}=0.6$.

To put the model into practice, we deduce from (4) the internal reflectance spectrum $r_{g}$ of a blank paper by setting the ink coverage

1. For a $45 / 0$ degrees measuring geometry, we set $K=0$.

2. The internal transmittance $t_{w}$ of white (no ink) is 1 at each wavelength $a=0 . R_{w}$ is the measured blank paper reflectance.

$$
r_{g}=\frac{R_{w}-K \cdot r_{s}}{1+(1-K) \cdot r_{i} \cdot r_{s}+r_{i} \cdot R_{w}-r_{s}-r_{i}}
$$

We then extract the transmittance of the individual inks and ink combinations $t_{w}, t_{c}, t_{m}, t_{y}, t_{r}, t_{g}, t_{b}, t_{k}$ by inserting in eq. 4 as $R(\lambda)$ the measured solid (100\%) ink coverage reflectance $R_{i}$ and by setting the ink coverage $a=1$.

$$
t_{i}=\sqrt{\frac{R_{i}-K \cdot r_{s}}{r_{g} \cdot r_{i} \cdot\left(R_{i}-K \cdot r_{s}\right)+r_{g} \cdot\left(1-r_{i}\right) \cdot\left(1-r_{s}\right)}}
$$

We must also take a possible physical dot gain into account. For each ink, we fit according to Clapper-Yule (eq. 4) the unknown physical coverages of the measured single ink patches at nominal coverages of $10 \%, 20 \%, \ldots 90 \%$ by minimizing the sum of square differences between measured spectra and predicted spectra. For the basic Clapper-Yule model, fitted single wedge cyan, magenta and yellow surface coverages are lower than the nominal surface coverages, i.e. we obtain a negative dot gain. This is due to the fact that spectra predicted by the Clapper Yule model are darker than the corresponding measured spectra. The fitted negative dot gain tends to bring both spectra to the same levels, i.e. the predicted and measured spectra intersect each other (Figure 2).

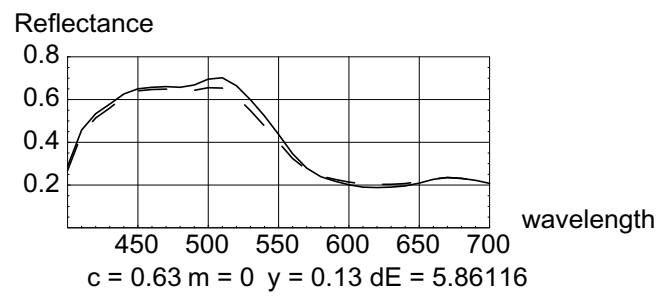

Figure 2: Example of measured (continuous) and predicted (dashed) reflection spectra according to the Clapper-Yule prediction model, with negative dot gain

Spectra predicted by the Clapper-Yule model (without the negative dot gain compensation) are too dark, because according to the measured modulation transfer function of paper [Inoue et. al. 1997], light does not travel significantly more than $1 / 10$ of a $\mathrm{mm}$ within coated paper. With a screen frequency of 60 lines per $\mathrm{cm}(150$ lines per inch), the probability that light having entered at a position having a certain ink color exits from a position of the same color is higher than the coverage of that ink color. Therefore, the basic assumption of the Clapper-Yule model, i.e. the probability of light exiting from a specific colorant being equal to that colorant coverage, is not fulfilled.

In order to set a base line for improvements, the accuracy of the basic Clapper-Yule model including physical dot gain is tested for a set of 729 patches produced by generating all combinations of inks at nominal coverages $0 \%, 12.5 \%, 25 \%, 37.5 \%, 50 \%, 62.5 \%$, $75 \%, 87.5 \%$ and $100 \%$. Measured and predicted spectra are converted to CIE-LAB values and the resulting error is computed. For the Clapper-Yule model, a mean error of $\Delta E=5.37$ was obtained, the maximal error is 12.1 and 577 values have a $\Delta E$ greater than 4 .

In order to enhance the basic Clapper-Yule model, we assume that a certain part $b$ of the incident light through a given colorant is reflected back and exits from the same colorant. The part (1-b) of the incoming light behaves in the same way as in the basic Clapper-Yule model described above. We also make the simplifying assumption that the part $b$ of the incident light which is reflected onto the same colorant also exits from the same colorant after one or several reflections at the coated paper-air interface.

Taking again multiple reflections into account, the attenuation of the part of the incoming light exiting from the same ink color 
(either no ink or ink with coverage $a$ ) at the first exit is

$$
\left(1-r_{s}\right) \cdot r_{g} \cdot\left(1-r_{i}\right) \cdot\left(1-a+a \cdot t^{2}\right)
$$

at the $2^{\text {nd }}$ exit, the attenuation is

$$
\left(1-r_{s}\right) \cdot r_{g} \cdot\left(1-r_{i}\right) \cdot\left(r_{g} \cdot r_{i}\right) \cdot\left(1-a+a \cdot t^{4}\right)
$$

and at the $\mathrm{n}^{\text {th }}$ exit the attenuation is

$$
\left(1-r_{s}\right) \cdot r_{g} \cdot\left(1-r_{i}\right) \cdot\left(r_{g}{ }^{n-1} \cdot r_{i}^{n-1}\right)\left[(1-a)+a \cdot t^{2 n}\right]
$$

The sum of all light that exits after an infinite number of reflections yields the spectrum

$$
R(\lambda)=\left(1-r_{s}\right) \cdot\left(1-r_{i}\right) \cdot\left(\frac{(1-a) \cdot r_{g}}{1-\left(r_{i} \cdot r_{g}\right)}+\frac{a \cdot r_{g} \cdot t^{2}}{1-\left(r_{i} \cdot r_{g} \cdot t^{2}\right)}\right)
$$

Equation (8) reflects the application of the Saunderson [1942] correction accounting for multiple internal reflections at the paper-air interface: the first term models the paper without ink (internal reflectance $r_{g}$ ) and the second term the paper printed with solid ink (internal reflectance $r_{g} \cdot t^{2}$ ).

The enhanced model (eq. 9) comprises a part $b$ of light propagated along short and middle distances (eq. 8) and a part (1-b) of the light propagated along long distances (eq. 5).

$$
R(\lambda)=K \cdot r_{s}+\left(1-r_{s}\right) \cdot r_{g}\left(1-r_{i}\right) \cdot\left[b \cdot\left[\sum_{j=1}^{8} \frac{a_{j} \cdot t_{j}^{2}}{1-r_{i} \cdot r_{g} \cdot t_{j}^{2}}\right]+(1-b) \frac{\left(\sum_{j=1}^{8} a_{j} \cdot t_{j}\right)^{2}}{1-r_{g} \cdot r_{i} \cdot \sum_{j=1}^{8} a_{j} \cdot t_{j}^{2}}\right]
$$

When fitting simultaneously the spectra of single ink coverages (dot gain of individual inks) and the parameter $b$ (ratio of the incident light reflected back to the same colorant), one obtains values of $b$ between 0.45 and 0.7 . For standard offset printing, i.e. screens mutually rotated by $30^{\circ}$ and a screen frequency of 150 lines (screen elements) per inch, the fraction $b$ of the light exiting from the same color as the incoming light yielding the smallest mean error for all 729 test patches is $b=0.6$.

In offset printing, trapping, i.e. the decrease in thickness of ink layers when two or more inks are printed one on top of another is generally considered to be a problem [Kipphan 2001, pp. 103-105], [Stanton and Raencic 2001]. Luckily, our model automatically takes care of trapping between standard inks by computing the internal transmittances of the red, green, blue and black colorants according to equation (7). However, we observed an ink spreading phenomenon when a second ink is printed over a first ink or when a third ink is printed on top of two inks. In the same way as physical dot gain, ink spreading enlarges the surface of a printed dot and tends to lower the resulting reflection spectrum, i.e. it yields slightly darker colors (Figure 3).

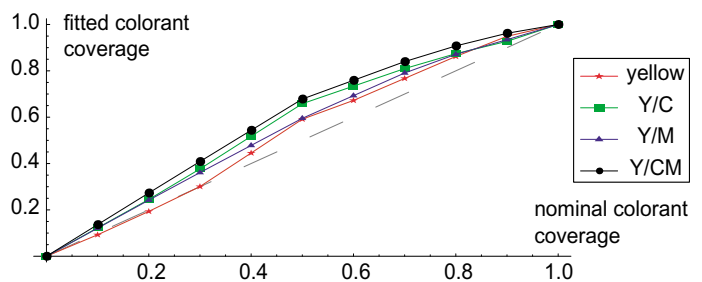

Figure 3: Tone reproduction curves for yellow alone, yellow over solid cyan, yellow over solid magenta and yellow over solid cyan and magenta

Since, in the considered printing process, offset inks are printed by first depositing cyan, then magenta and then yellow, we fit the respective ink spreaded coverages of magenta over solid (i.e. $100 \%)$ cyan $f_{m, c}(m)$, yellow over solid cyan $f_{y, c}(y)$, yellow over solid magenta $f_{y, m}(y)$ and yellow over solid cyan and magenta $f_{y, c m}(y)$ by minimizing the square differences between measured spectra and spectra predicted according to equation (9).

In front of the enhanced Clapper-Yule model, we therefore introduce a stage taking into account dot gain and ink spreading (Figure 4). An input nominal color with coverages cmy is converted to an intermediate color with coverages c' $m$ ' $y$ ' obtained as a weighted sum of dot gain and ink spreading contributions.

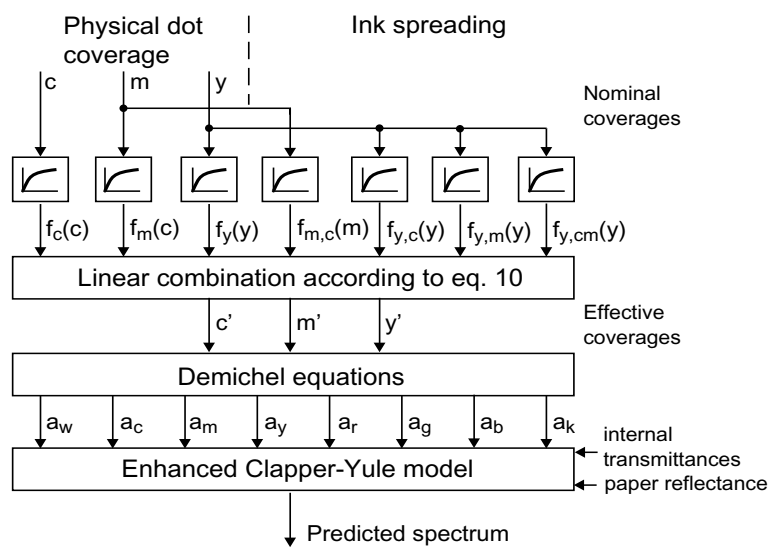

Figure 4: Enhanced Clapper-Yule model taking into account dot gain and ink spreading

The weighting coefficients are given by the respective effective colorant coverages.

$$
\begin{aligned}
& c^{\prime}=f_{c}(c) \quad ; \text { cyan first ink } \\
& m^{\prime}=f_{m}(m)\left(1-c^{\prime}\right)+f_{m, c}(m) c^{\prime} ; \text { magenta alone and blue } \\
& y^{\prime}=f_{y}(y)\left(1-c^{\prime}\right)\left(1-m^{\prime}\right) \quad ; \text { yellow alone, red, green and black } \\
& \quad+f_{y, m}(y)\left(1-c^{\prime}\right) m^{\prime}+f_{y, c}(y) c^{\prime}\left(1-m^{\prime}\right)+f_{y, c m}(y) c^{\prime} m^{\prime}
\end{aligned}
$$

With the enhanced Clapper-Yule model and by taking ink spreading into account, a mean error between predicted and measured spectra ( 729 spectra) of $\Delta E=2.36$ was obtained, the maximal error is $\Delta E=7.09$ and only 64 values have a $\Delta E$ greater than 4 . In contrast to Stollnitz's model [Stollnitz et al. 1998], our model only fits dot sizes when printed alone or in combination with other inks (physical dot gain, ink spreading), but predicts spectra (36 components). It does not fit the spectra of any of the inks or of paper. All internal spectra are calculated from measured spectra, according to the model. Therefore the model reflects at least to a certain extent the underlying physical phenomena.

\section{A prediction model for standard inks printed on top of a metallic inks}

Metallic inks are generally formed by flat opaque metal (aluminium) particles floating within a liquid binder. The particles tend to align themselves horizontally along the surface of the ink film (leafing effect). A higher leafing effect yields a more metallic looking ink surface [Kern et. al. 1991]. As is the case for metallic surfaces [Cook and Torrance 1982], a coated paper printed with a metallic ink reflects a significant part of the incoming light in and around the specular direction, and an additional part of the light diffusely in all directions.

According to the concentration of the metallic particles, the metallic ink layer also absorbs part of the incoming light flux and transmits another part of the incoming light flux onto the underlying substrate (coated paper). Due to the fact that the metallic particles float within the varnish, light reflected by theses particles is also partly, at the varnish-air interface, reflected back into the metallic layer, yielding diffuse multiple reflections. We would like to predict the reflection spectra of standard inks $\left(c_{s} m_{s} y_{s}\right)$ printed on top of a metallic ink (silver $s$ ), when measured under non-specular reflection conditions. Fortunately, standard photospectrometers have a geometry of $45 / 0$ degrees, i.e. the incident angle is 45 
degrees and the exit angle is 0 degree. Spectrometric reflection measurements (Figure 6) show that, compared with an ideal diffuse reflector, $26 \%$ to $27 \%$ of the light is reflected by a solid metallic ink printed on a coated paper at $0^{\circ}$ exit angle (similar values for all wavelengths). In a first approximation, for the $45^{\circ} / 0^{\circ}$ measuring geometry, we model the two phenomena, i.e. the decrease in reflected light by metallic particles due to the $0^{\circ}$ exiting angle and the absorption of light traversing the metallic layer by an equivalent ink layer having a specific internal transmittance. Its transmittance $t_{s}$ is obtained by measuring the reflectance $R_{s}$ of a solid metallic (silver) ink patch and inserting it into equation (7). From a strict point of view, the equivalent ink layer transmittance is only valid for the current measuring geometry. This means that the prediction model for colors printed with a metallic ink is only valid for "standard" non-specular reflection angles, i.e. angles close to the incident and exiting angles of the measuring instrument, in our case $45^{\circ} / 0^{\circ}$. At strongly non-specular reflection angles, where the amount of specularly reflected light is significantly lower, colors printed with a metallic ink have a darker appearance than in the standard non-specular case.

Patches printed with one or several inks over a solid silver ink show a strong trapping phenomenon, i.e. only a fraction of the ink that should be printed is effectively printed on top of a metallic ink. To predict the reflection spectra of standard inks printed over a metallic ink, we extend the enhanced Clapper-Yule model described in Section 3 by including the equivalent internal transmittance $t_{s}$ of the metallic ink layer into equation (9) and by taking trapping into account. In equation (9), since standard inks are transparent, instead of individual colorant transmittances $t_{j}$, we use transmittances $t_{s j}=t_{s} \cdot t_{j}$. Trapping is taken into account by replacing the ink spreading and dot gain effects by the trapping effect.

\section{Modeling the trapping effect}

In the case of printing a standard ink on top of a metallic ink, trapping is mainly due to the poor adherence of the standard ink. Only a portion of the standard ink is transferred onto the metallic ink layer. Parts of the metallic ink layer remain uncovered. When a second standard ink is printed on top of a first standard ink over a metallic ink, trapping further decreases the coverage of the second ink. At the same time, the first standard ink may spread out, possibly due to the better adherence properties of the second ink or to the pressure produced when printing the second ink layer. Therefore the observed surface coverages represent trapping and also to a certain extent, ink spreading.

The approach we follow in order to take trapping into account when printing on top of a metallic ink is to first fit the surface coverages of each standard ink alone. We obtain the trapping coverage curves $h_{c}(c), h_{m}(m), h_{y}(y)$ for respectively the cyan, magenta and yellow single inks printed over the metallic ink (red curves, Fig. 5). We then fit the surface coverage of each ink superposed with one of the other solid inks (green and blue curves in Fig. 5). This yields 6 trapping coverage curves: the trapping of magenta superposed with solid yellow $h_{m, y}(m)$, of yellow superposed with solid magenta $h_{y, m}(y)$, of cyan superposed with solid yellow $h_{c, y}(c)$, of yellow superposed with solid cyan $h_{y, c}(y)$, of cyan superposed with solid magenta $h_{c, m}(c)$ and of magenta superposed with solid cyan $h_{m, c}(m)$. Finally, we fit the individual trapping coverages of one ink printed in superposition with two solid inks (purple curves, Figure 5). We obtain three trapping coverage curves for respectively the cyan, magenta and yellow coverages in combination with the two other solid inks, i.e. $h_{c, m y}(c), h_{m, c y}(m), h_{y, c m}(y)$. All fitting operations are carried out with the enhanced Clapper-Yule model extended to metallic ink by minimizing the sum of square differences between predicted and measured spectra.

The trapped ink coverages are illustrated in Figure 5. Input values are nominal ink coverages and output values are the fitted trapped ink coverages. Due to trapping, the ink coverage tends to decrease when increasing the number of superposed inks. However, when a second ink is printed on top of a first ink, due to its improved adherence or pressure, there is also a tendency to increase the coverage of the first ink. In the present case, cyan overprinted by yellow tends to have a slightly larger coverage than cyan alone (Fig. 5a). We have a strongest decrease in individual ink coverages when three inks are superposed. The order in which the inks are printed is important. The ink coverage for cyan (first ink) alone or in combination with a second ink is increased (Fig. 5a). When magenta (2nd ink) is printed on top of cyan it exhibits a stronger ink reduction than when printed alone (Fig. 5b). For yellow, this phenomenon is even more pronounced: yellow (3rd ink) printed on top of magenta or cyan has a considerable coverage reduction and on top of both cyan and magenta inks has the largest coverage reduction (Fig. $5 \mathrm{c}$ ).

a)

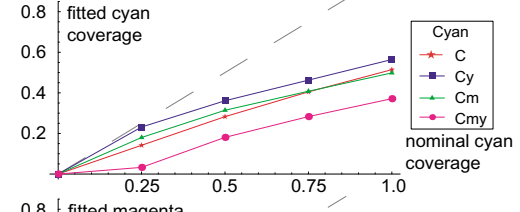

b)

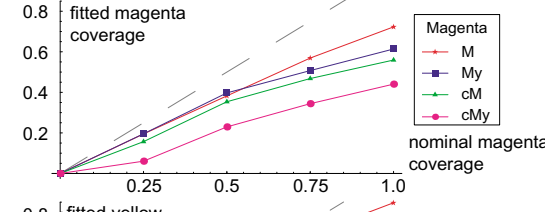

c)

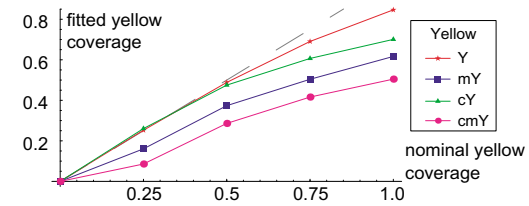

Figure 5: Reduced surface coverage values of individual inks when printed alone or in superposition with one or two solid standard inks printed on top of a solid metallic ink

The equations (11) allow us to compute trapped coverage values by weighting the contributions of the trapped coverages of a single ink, of one ink superposed with one solid ink and of one ink superposed with two solid inks. Weighting factors are established by reasoning in similar terms as for establishing the Demichel equations (eq. 2), i.e. by considering nominal $(c, m, y)$ and trapped $\left(c_{t}, m_{t}, y_{t}\right)$ ink coverages. Trapped ink coverages have been fitted for nominal coverages of the considered inks (one ink alone, one ink superposed with one or two solid inks). Therefore, the weight of the trapping coverage $h_{i, j}(i)$ of one ink $i$ superposed with a second ink $j$ is proportional to the nominal coverage $c_{j}$ of that ink. Furthermore, it is proportional to the amount of the available effective surface, i.e. one minus the trapping (effective) coverage of a noncontributing ink. Weighting factors are normalized so as to ensure that their sum is one. We obtain equations (11).

$$
\begin{aligned}
c_{t}= & {\left[h_{c}(c) \cdot\left(1-m_{t}\right) \cdot\left(1-y_{t}\right)+h_{c, m}(c) \cdot m \cdot\left(1-y_{t}\right)\right] } \\
& \left.+h_{c, y}(c) \cdot y \cdot\left(1-m_{t}\right) \cdot+h_{c, m y}(c) \cdot m \cdot y\right] / p_{c} \\
m_{t}= & {\left[\left(h_{m}(m) \cdot\left(1-c_{t}\right) \cdot\left(1-y_{t}\right)+h_{m, c}(m) \cdot c \cdot\left(1-y_{t}\right)\right]\right.} \\
& \left.+h_{m, y}(m) \cdot y \cdot\left(1-c_{t}\right)+h_{m, c y}(m) \cdot c \cdot y\right] / p_{m} \\
y_{t}= & {\left[h_{y}(y) \cdot\left(1-c_{t}\right) \cdot\left(1-m_{t}\right)+h_{y, c}(y) \cdot c \cdot\left(1-m_{t}\right)\right] } \\
& \left.+h_{y, m}(y) \cdot m \cdot\left(1-c_{t}\right)+\cdot h_{y, c m}(y) \cdot c \cdot m\right] / p_{y}
\end{aligned}
$$

The normalizing factors $p_{c}, p_{m}$ and $p_{y}$ are given by the sum of the weighting coefficients, i.e. for inks $i, j, k$ with nominal coverages $c_{i}, c_{j}$ and $c_{k}$ and trapped coverages $c_{i t}, c_{j t}$ and $c_{k t}$

$$
p_{i}=\left(1-c_{j t}\right) \cdot\left(1-c_{k t}\right)+c_{j} \cdot\left(1-c_{k t}\right)+c_{k} \cdot\left(1-c_{j t}\right)+c_{j} \cdot c_{k}
$$

One obtains the trapped coverages values $c_{t}, m_{t}, y_{t}$ by applying equations (11) iteratively, with starting values of the trapped ink coverage values equal to the nominal coverage values. The system converges after 4 iterations. These equations represent a relatively coarse approximation of the complex phenomenon of printing with several standard inks on top of a metallic ink. Nevertheless, they yield good results (see Appendix, Table A1). 


\section{Raised metallic reflection spectrum}

Taking into account trapping is not yet sufficient for building an accurate prediction model. A detailed analysis of the spectra of colors printed on top of a metallic ink reveals that the ink printed on top of the metallic ink has the effect of raising the metallic ink's non-specular reflection values. For example, the measured reflectance of solid magenta and solid yellow printed on top of the solid silver ink is higher in the red part of the spectrum than the reflection spectrum of silver alone (Fig. 6).

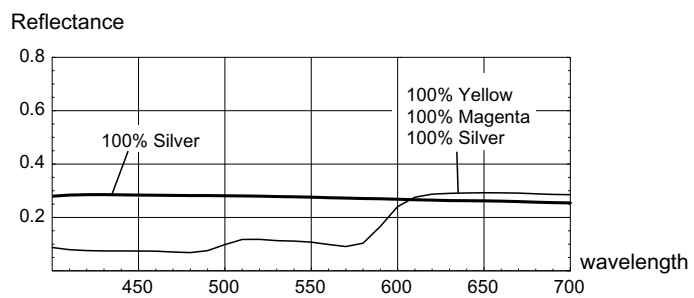

Figure 6: Reflection spectra of silver alone and solid magenta + solid yellow printed on top of solid silver

We suspect that the raised metallic reflection spectrum is induced by one or several of the following phenomena:

- the ink deposited on top of the metallic ink forms ink droplets whose surface curvature modify the light's refraction and reflection angles and allow more light to be reflected by the metallic particles at the zero degree exit angle,

- the ink pigments scatter back a small part of the incoming light,

- printing an ink on top of silver modifies the leafing effect and enhances the reflection of the metallic ink layer at the 0 degree exit angle.

The detailed analysis of these phenomena is beyond the scope of the present paper. We simply model the raised metallic reflection spectrum by a scalar raising factor. Metallic ink reflection spectra raising factors are obtained by fitting them at the same time as fitting the reduced surface coverages due to trapping for one solid ink and for the superpositions of two solid inks. Since the superposition of 3 solid inks (black) absorbs light in all spectral bands, the metallic reflection spectrum does not need to be raised. When printing with solid cyan, magenta and yellow on top of silver, one obtains raising factors of respectively $f_{r c}=1.069, f_{r m}=1.15$, $f_{r y}=1.16$. When printing with solid magenta and yellow (red), cyan and yellow (green) and cyan and magenta (blue) on top of silver, one obtains fitted raising factors of respectively $f_{r r}=1.25, f_{r g}=1.47$ and $f_{r b}=1.31$. For the metallic ink alone and for the superposition of solid cyan, magenta and yellow (black), the metallic reflection spectrum is not raised, i.e. $f_{r w}=1$ and $f_{r k}=1$. The raising factor $f_{R}$ (Fig. 7) is a weighted mean of the individual raising factors, the weights being given by the nominal amounts of colorants (eq. 2). This raising factor $f_{R}$ raises the initially measured metallic ink reflection spectrum $\left(45^{\circ} / 0^{\circ}\right.$ measurement geometry), from which the equivalent transmission spectrum $t_{s}$ of the metallic ink is computed according to equation (7). The resulting complete prediction model for predicting the spectra of inks printed over a metallic ink is shown in Figure 7.

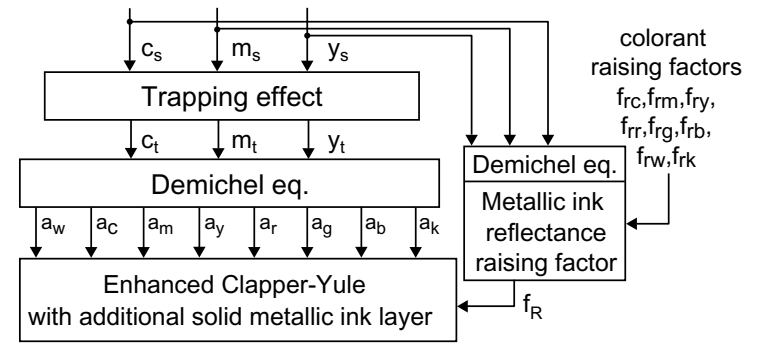

Figure 7: The elements of the prediction model for predicting the spectra of inks printed on top of a solid metallic ink
For predicting the spectrum of a patch printed with an effective surface coverage $s$ of a metallic (silver) ink, we compute the part $s$ of the spectrum according to the prediction model introduced in the present section taking trapping and enhanced metallic ink reflection spectrum into account and the part (1-s) according to the enhanced Clapper-Yule prediction model introduced in section 3.

$$
R_{\text {tot }}(\lambda)=s \cdot R_{\text {scmy }}(\lambda)+(1-s) \cdot R_{c m y}(\lambda)
$$

In order to take into account the dot gain of silver, the effective surface coverage $s$ of the metallic ink is fitted according to equation (13), for the case of nominal coverages $s_{\text {in }}$ of $10 \%, 20 \%, 30 \%$, ..., $100 \%$ of the silver ink alone, printed over coated paper. This yields the silver surface coverage curve $s\left(s_{i n}\right)$.

The enhanced Clapper-Yule model, together with trapping and metallic ink spectrum raising corrections allow us to accurately predict the color of a patch printed with freely chosen coverages of cyan, magenta, yellow and up to $100 \%$ silver. Our test set was produced with variations of cyan, magenta, yellow inks at $0 \%, 25 \%$, $50 \%, 75 \%$ and $100 \%$ nominal coverages and the silver ink at $10 \%$, $30 \%, 50 \%, 80 \%$ and $100 \%$ yielding 625 samples. The comparison between predicted and measured spectra (Table A1 in the Appendix) yields excellent results, similar to the results obtained when predicting the spectra of standard inks (mean prediction error $\Delta E=2.37)$

\section{Calibrating the metallic ink printing process}

We need to match the color of patches printed with standard inks (cmy coverages) by colors printed by the superposition of the metallic ink and standard inks ( $s c_{s} m_{s} y_{s}$ coverages). With our prediction model for standard inks, we may predict the reflection spectrum of patches printed with nominal coverages of standard inks. With the model for predicting the spectrum of colors printed with a metallic ink (eq. 11), we may then fit corresponding $s c_{s} m_{s} y_{s}$ coverages by minimizing the sum of square differences between the reflection spectrum predicted for the standard ink patch and the reflection spectrum predicted for the metallic ink patch.

We construct for the desired coverage of the metallic ink a rectilinear 3D grid (lookup table) in the cyan, magenta, yellow output color space by considering for example nominal cmy input surface coverages of $0 \%, 12.5 \%, 25 \%, 37.5 \%, 50 \%, 62.5 \%, 75 \%, 87.5 \%$ and $100 \%$. Each grid point associates to nominal cmy coverages corresponding $s c_{s} m_{s} y_{s}$ coverages incorporating the metallic ink. With a C++ program implementing Powell's function minimization method [Press et. al. 1992, section 10.5, pp. 412-420], the creation of the 3D lookup table takes 65 minutes on a $600 \mathrm{MHz}$ Pentium III computer.

When replacing standard inks by the superposition of a metallic ink and standard inks, we cannot impose the surface coverage of the metallic ink. We may ask for a given silver surface coverage $s$, and the system will try to find a solution by fixing $s$ and trying to fit the $c_{s} m_{s} y_{s}$ coverages so as the minimize the sum of square differences between the predicted $s c_{s} m_{s} y_{s}$ reflectance spectrum and the reflectance spectrum predicted for the input cmy values. However, in highlight tones, it is impossible to ask for a high coverage of silver, i.e. the fitting process may not return a valid solution. In these cases, the required coverage $s$ of silver is successively lowered, for example in steps of 5\%, until a possible solution is found.

In order to hide within an image patterns at non-specular observation angles, which are to be revealed at specular reflection angles, a colorimetric difference value of $\Delta E<2$ between patches printed with standard inks and corresponding patches printed with metallic inks is required. To allow for a sufficient metallic ink coverage, patterns should therefore preferably be hidden in darker image areas.

Synthesizing images with embedded metallic microstructures (Fig. 8) is less critical. In the case that all the microstructure foreground is rendered with a metallic ink, a significant color deviation 
between predicted and measured metallic colors (e.g. $\Delta E=4$ ) may be tolerated.

Once the 3D lookup table is created, the program which synthesizes the hidden metallic ink patterns receives as input a mask specifying the areas to be printed with metallic ink. Initial cmy pixels located within the mask are converted by tri-linear interpolation with the help of the lookup table into $s c_{s} m_{s} y_{s}$ coverage values. Input pixels located outside the mask are simply transferred as they are to the output color coverages, i.e. $c_{s}=c, m_{s}=m, y_{s}=y$. The color output layers are halftoned in the same way as for standard offset printing, with screen frequencies of $150 \mathrm{lpi}$ and standard screen angles $15,45,75$ and 0 degrees for respectively the cyan, silver, magenta and yellow layers.

\section{Design Examples}

In the first category of design examples, we would like to enhance the reflectance of microstructure patterns embedded into a color image at specular reflection angles. Figure 8 shows a sample bank note dedicated to Professor Auguste Piccard, who, in 1931, was the first man to explore the stratosphere in a balloon specially conceived by him.

Piccard's portrait is initially given by a grayscale image to be rendered by combining a single Pantone color and the paper white. Piccard's portrait is reproduced with two superposed image layers. A first image layer renders the portrait with microletters at high intensity levels and standard clustered dot halftones at middle and low intensity levels. A second image layer reproduces the portrait with warped microstructure shapes representing the "SSP" logo. This second layer is rendered thanks to artistic screening, a method for creating images by carefully designed microstructure shapes, which change according to the local image intensity level [Ostromoukhov and Hersch 1995]. The foreground of this second layer incorporating the microstructure shapes (letters) is rendered with a silver ink at $70 \%$ coverage and with coverages of cyan, magenta, and yellow matching at non-specular angles the cmy color separations of the selected Pantone color.

When viewed from a large distance at non-specular viewing angles, mainly the portrait is visible. By reducing the viewing distance, the microstructure patterns "SSP" become apparent. When seen from nearby under specular light reflection (e.g. by tilting the picture), the microstructure printed with the metallic ink becomes strongly visible. Its high contrast with the background tends to mask all other image elements. Only the outline of the face rendered with the metallic ink microstructure remains visible. This very striking effect may be used as a visual authentication feature. Since photocopiers are not capable of reproducing images with metallic inks, the microstructure enhancement effect at specular viewing angles is lost in photocopied documents.

The second category of design examples relies on the inversion effect. A pattern printed with metallic inks is dark at strongly nonspecular reflection angles and highlight at specular reflection angles. With metallic ink microstructures embedded into images, the transition between dark and highlight microstructures may produce interesting effects. Figure 9 shows the image of a Masai fighter embedded into a color wedge. By tilting the figure, the inversion effect modifies the fighter image: the image is first dark, then partially hidden within the color wedge and finally appears at a specular viewing angle as highlight. To produce the picture, we first halftone the image of the Masai fighter with a dither matrix incorporating the microstructure pattern. The resulting halftoned binary image is then used as a mask for rendering the color wedge by combining the metallic ink and the standard inks.

A further use of the inversion effect is shown in Figure 10. This example shows a warped staircase within a stylized shield. At non specular angles, the depth effect is not easily perceived whereas at specular angles, the staircase becomes immediately apparent. One can see at the top, bottom, left and right parts of the shield dark brown surfaces. At specular reflection angles, the high reflectance of the metallic ink creates a strong contrast masking the dark brown surfaces and letting the staircase appear without discontinuity.

In the last category of design effects, we try to hide a ghost pattern at non-specular reflection angles and make it apparent at specular reflection angles. Completely hiding a pattern at standard nonspecular reflection angles (in our case $45^{\circ} / 0^{\circ}$ ) requires that the prediction models presented in sections 3 and 4 work at a high accuracy: colors printed with a metallic ink should be less than $\Delta E=2$ apart from the corresponding colors printed with standard inks. Since the accuracy of the present printing press (Komori Lithrone 26) on a well controlled printing run is around $\Delta E=2$ within a page and $\Delta E=3$ across pages, we can only to a certain extend try to hide patterns.

Figure 11 shows a ghost pattern hidden within the African mask. The ghost pattern becomes apparent under a specular observation angle and allows to verify the authenticity of a security document. Since an accurate prediction model as well as accurate printing presses are necessary for hiding the metallic ink ghost pattern within a color image, metallic ghost patterns offer a significant anti-counterfeiting protection.

\section{Conclusions}

Embedding metallic patterns within color images offers new design capabilities for artists trying to create striking effects of a high aesthetic quality. In order to be able to embed patterns printed with a metallic ink within a color image, we propose an accurate model for printing both with standard inks and with a combination of a metallic ink and of standard inks. The model we propose is an enhancement of the classical Clapper-Yule model taking into account the fact that proportionally more incident light through a given colorant surface is reflected back onto the same colorant surface than onto other colorant surfaces. Since the model incorporates the effects of light scattering and multiple reflections within halftone layers, its coverage predictions include optical dot gain. Physical dot gain is separately modeled by fitting the coverages of each ink according to the enhanced Clapper-Yule model. We also model ink spreading, i.e. the physical dot gain obtained when printing one ink over another ink.

The second model we propose aims at predicting reflection spectra of patches printed with a metallic ink and standard inks, for a 45/0 degrees measuring geometry. The metallic ink comprises a certain concentration of small flat metallic particles aligned along the surface of the ink. The part of the light which is directly reflected by the metallic surfaces is mostly specular and decreases towards non-specular exiting angles. For a $45^{\circ} / 0^{\circ}$ measuring geometry, we model both phenomena, i.e. the light reflected by the metallic particles and the light transmitted to the substrate below the ink layer and reflected back from the paper bulk by an equivalent solid ink layer with a specific internal transmittance.

Since the standard cyan, magenta and yellow inks do not adhere well on the metallic ink, there is a strong trapping effect. Trapping induces a significant reduction in surface coverage. The amount of surface coverage reduction is specific for each ink and combination of inks. We also observed that printing a standard ink over a metallic ink raises the metallic ink reflection spectrum. This effect is taken into consideration by introducing metallic ink reflection spectrum raising factors specific for each ink and superposition of inks. Surprisingly, our full model for printing with a combination of a metallic and standard inks is as accurate as the model for printing with standard inks.

Since our models only fit surface coverages when inks are printed alone or in superposition with other inks (dot size, ink spreading, trapping), but predict spectra (36 components), they reflect at least to a certain extent the underlying physical phenomena.

Hiding metallic patterns into printed images requires the full accuracy offered by our prediction models. Therefore they may be 
correctly printed only on high-end well-tuned offset presses. However, the other proposed metallic ink effects such as lightness inversion and the enhancement of slightly apparent microstructure patterns require less precision and can therefore be attained with standard offset presses. These effects already prevent security documents from being counterfeited by simple means such as color photocopy.

The model we propose is according to our knowledge the first model able to predict the reflection spectra of prints combining a metallic ink and standard inks. It opens the way towards color separation with metallic inks and the creation of high-end designs incorporating metallic effects within printed images.

The presented results are only a first step towards the creation of images integrating metallic and standard inks. There are several ways of extending this research. One may try to incorporate into printed images not only a single silver ink, but also several colored metallic inks (e.g. inks from the metallic ink Pantone catalogue). It would also be interesting to verify if the interaction of light and patches printed with a combination of a metallic and of standard inks, for any combination of illumination and observation angles can be described by physical reflection models such as [Cook and Torrance, 1982], [He et. al. 1991], [Ershov et. al. 2001] or by an empirical model [Ward 1992]. Such a deeper insight may allow to create previewing software enabling designers to preview the results of their work before printing.

\section{Acknowledgements}

The authors would like to thank the designers who created the sample bank notes. Bertrand Bender designed the Auguste Piccard banknote (Fig. 8). Pascal Fehr and Ingo Baumgartner designed the African banknotes (Figs. 10 and 11). We further thank Nicolas Rudaz who created the microstructures of Figs. 8 and 10 and Edouard Forler who created the masks allowing to embed the Masai fighter image into a wedge (Fig. 9). We also thank Mathieu Hebert and Greg Ward for helpful discussions on phenomena related to the reflectance of paper printed with the superposition of a metallic ink and standard inks. Finally, we thank Sergio Lizzola of SSP SA, a startup company specialized in security printing, which printed the calibration patches, the test patches and the final designs. The project was partly financed by the Swiss CTI, contract 5018.1 and partly by the Swiss National Science Foundation, contract 2000-065167.01.

\section{Bibliography}

ClapPer, F.R. and Yule J.A.C 1953. The effect of multiple internal reflections on the densities of halftone prints on paper. Journal of the Optical Society of America, Vol. 43, 600-603.

CoOK, R.L. and Torrance, K.E. 1982. A Reflectance Model for Computer Graphics. ACM Trans. on Graphics, Vol. 1., Nol. 1, 7-24.

DeMichel, M.E. 1924. Procédé, Vol. 26, 17-21.

Ershov, S., Kolchin, K., and MyszKowski, K. 2001. Rendering Pearlescent Appearance Based on Paint-Composition Modelling. Proc. Eurographics 2001, special issue of Computer Graphics Forum, Vol. 20, No. 3.

He, X.D., Torrance, K.E., Sillion, F.X. and Greenberg, D.P. 1991. A Comprehensive Physical Model for Light Reflection. In Computer Graphics (Proc. of ACM SIGGRAPH'91), Vol. 25, No. 4, ACM, 175186.

Hecht, E. 1974. Schaum's Outline of Optics, Mc-Graw-Hill.

InOUe, S, Tsumara, N., and MiYAKe Y. 1997. Measuring MTF of Paper by Sinusoidal Test Pattern Projection. Journal of Imaging Science and Technology, Vol. 41, Nol. 6, 657-661.

JUDD, D.B. 1942. Fresnel reflection of diffusely incident light. Journal of Research of the National Bureau of Standards, Vol. 29, 329-332.

KANG, H.R. 1994. Applications of color mixing models to electronic printing. Journal of Electronic Imaging, Vol. 3, No. 3, 276-287.

KANG, H.R. 1997. Color Technology for Electronic Imaging Devices. SPIE Optical Engineering Press, 1997.
Kern, G.M., Micale, J., Lavelle, S. and Valenzuela P. 1991. Hiding power of aluminium pigments in printed ink films. American Ink Maker, vol 69 , no $10,60-68$.

KiPPHAN, H. 2001. Handbook of Print Media. Springer-Verlag.

KuBELKA P. 1954. New contributions to the optics of intensely light-scattering material, part II: Non-homogeneous layers. Journal of the Optical Society of America, Vol. 44, 330-335.

Neugebauer, H.E.J. 1937. Die theoretischen Grundlagen des Mehrfarbendrucks. Zeitschrift fuer wissenschaftliche Photographie, Vol. 36, 36-73, reprinted in Neugebauer Seminar on Color Reproduction, SPIE Vol1184, 1989, 194-202.

Ostromoukhov, V. and Hersch, R.D. 1995. Artistic Screening. Proc. of ACM SIGGRAPH 1995, ACM Press, Computer Graphics Proceedings, Annual Conference Series, 219-228, 1995

Press, W.H., Flannery, B.P., Teukolsky, S.A. and Fetterling, W.T. 1992. Numerical Recipes. Cambridge University Press, 2nd edition.

Rogers, G. 2000. A Generalized Clapper-Yule Model of Halftone Reflectance. Journal of Color Research and Application, Vol. 25, No. 6, 402407.

SAUNDERSON, J.L. 1942. Calculation of the color pigmented plastics. Journal of the Optical Society of America, Vol. 32, 727-736.

Stanton, A. and RaEncic, G. 2001. Ink Trapping and Colorimetric Variation. Proc. TAGA 2001, 258-281.

Stollnitz, E.J., Ostromoukhov, V. and SAlesin, D. 1998. Reproducing Color Images Using Custom Inks. Proc. of ACM SIGGRAPH 1998, ACM Press, in Computer Graphics Proceedings, Annual Conference Series, 267-274.

WARD, G. 1992. Measuring and modeling anisotropic reflection. In Computer Graphics (Proc. of ACM SIGGRAPH 92), Vol. 26, No. 2, 1267 274.

Williamson, J.S., Barnaby, G.V., and Doughty, G.V. 1994. US patent 5,370,976, Metallic Color Printing Process, issued Dec 6, 1994, see section "Background of the invention".

Wyble, D.R. and Berns, R.S. 2000. A Critical Review of Spectral Models Applied to Binary Color Printing. Journal of Color Research and Application, Vol. 25, No. 1, 4-19.

Yule, J.A.C. and NiELSEN, W.J. 1951. The penetration of light into paper and its effect on halftone reproductions.Proc. TAGA, Vol. 3,65-76.

\section{Appendix}

Table A1. Successive model improvements and corresponding prediction accuracies expressed in CIE-LAB $\Delta E$ values.

\begin{tabular}{c|c|c|c|c} 
& $\begin{array}{c}\text { Number } \\
\text { of samples }\end{array}$ & Max $\Delta E$ & $\begin{array}{c}\text { Mean } \\
\Delta E\end{array}$ & $\begin{array}{c}\text { \# samples } \\
\Delta E>\mathbf{4}\end{array}$ \\
\hline $\begin{array}{c}\text { Clapper-Yule with dot-gain } \\
\text { only }\end{array}$ & 729 & 12.09 & 5.37 & 577 \\
$\begin{array}{c}\text { Clapper-Yule, dot-gain and } \\
\text { ink spreading }\end{array}$ & 729 & 8.82 & 3.21 & 208 \\
$\begin{array}{c}\text { Enhanced Clapper-Yule } \\
\text { with dot-gain only }\end{array}$ & 729 & 7.09 & 2.94 & 118 \\
$\begin{array}{c}\text { Enhanced Clapper-Yule } \\
\text { with dot-gain and ink } \\
\text { spreading }\end{array}$ & 729 & 7.09 & 2.36 & 64 \\
$\begin{array}{c}\text { Metallic Clapper-Yule with } \\
\text { trapping effect }\end{array}$ & 625 & 7.49 & 3.11 & 145 \\
$\begin{array}{c}\text { Metallic Clapper-Yule with } \\
\text { trapping effect and with } \\
\text { raised reflection spectrum }\end{array}$ & 625 & 6.66 & 2.37 & 60
\end{tabular}




\section{Preview images for the electronic version}

Courtesy M. Monod and J. Turrian, students at EPFL

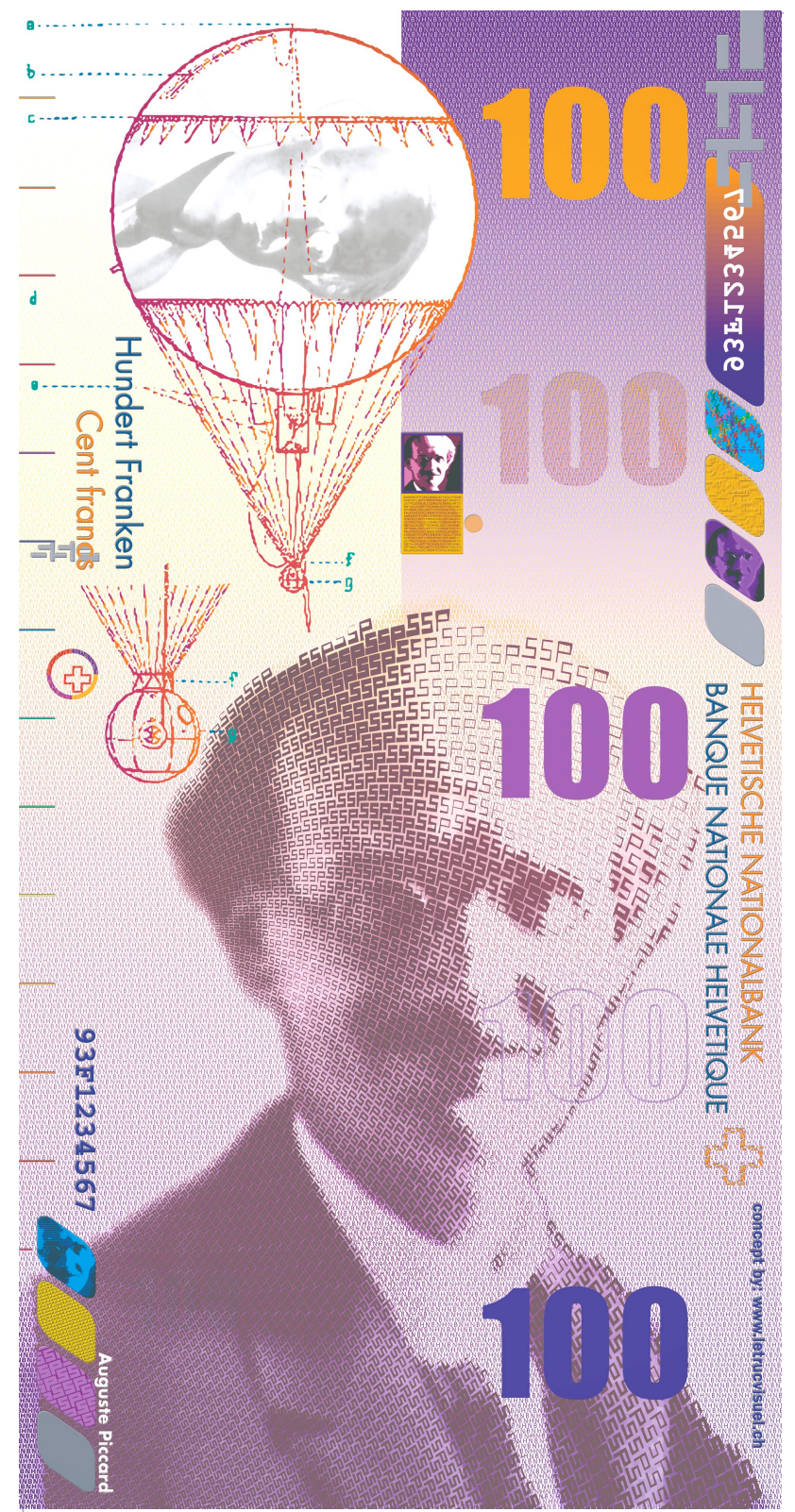

Figure 8: Banknote with the face of Auguste Piccard (preview image at a non-specular reflection angle, zoom-in for details).
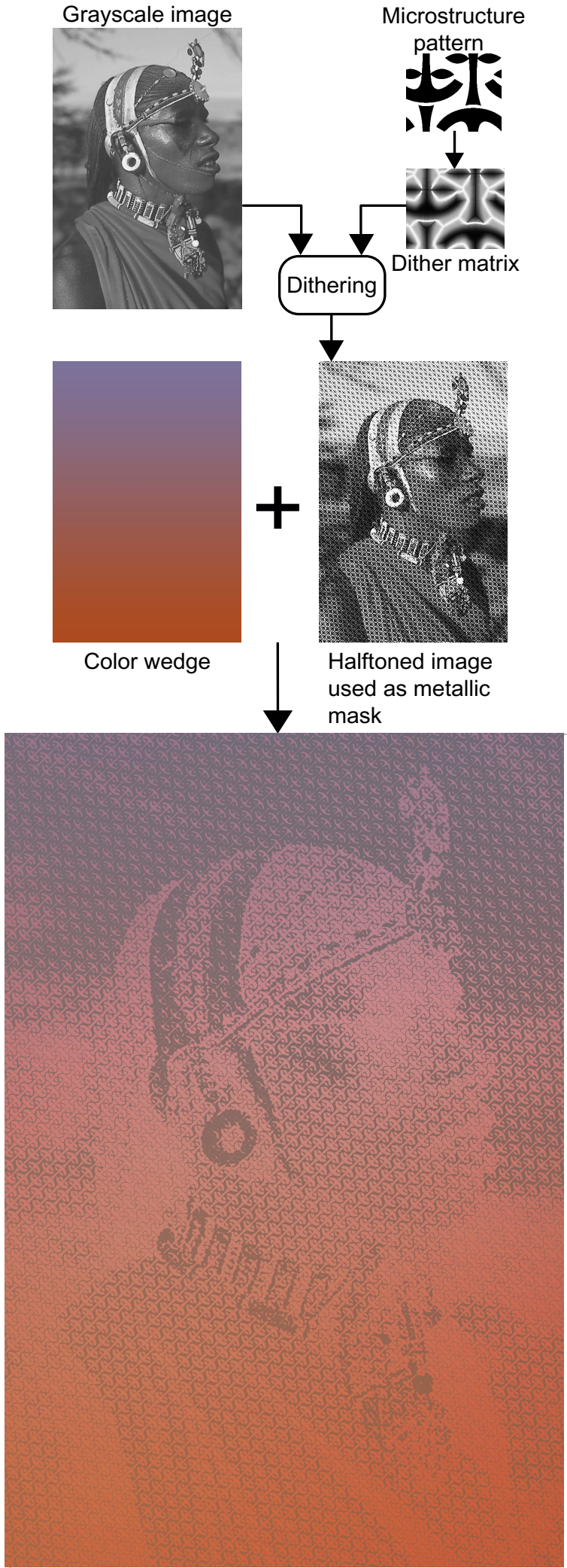

Figure 9: Preview image of a wedge embedding a Masai fighter image rendered with a metallic ink viewed under specular reflection. 


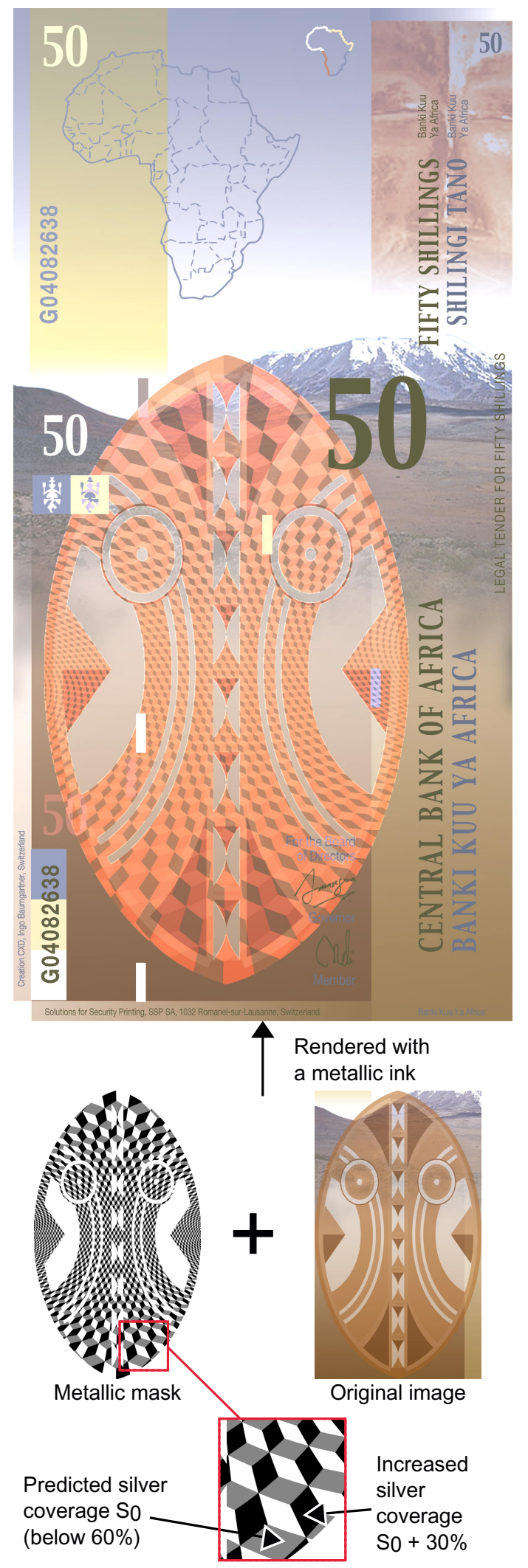

Figure 10: Preview image of a staircase becoming apparent under specular reflection thanks to the inversion effect.
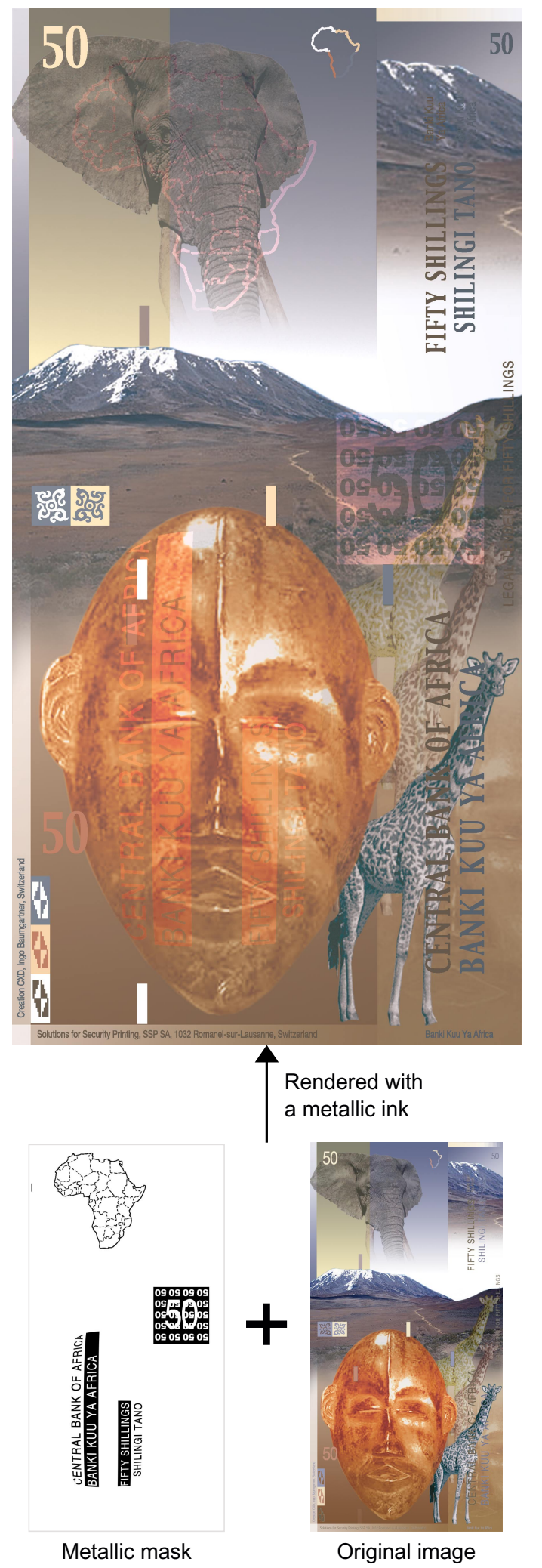

Figure 11: Preview image of a ghost pattern embedded within an African mask reproduced by the superposition of a metallic ink and standard inks (specular reflection). 